\title{
Energy management in a multi-building set-up via distributed stochastic optimization
}

\author{
Vedad Causevic, Alessandro Falsone, Daniele Ioli, Maria Prandini
}

\begin{abstract}
We address a cooling energy management problem in a multi-building setting where buildings need to maintain comfort conditions for the occupants by keeping their zones temperature within a certain range. To this purpose, each one of them has its own chiller and is connected to a shared cooling network. The goal is to minimize the overall district electricity cost over some finite time horizon by optimally setting the temperature set-points in the buildings and the energy exchange with the cooling network, compatibly with comfort and actuation constraints, while accounting for uncertainty, mainly due to outside temperature, people occupancy, and solar radiation. To this purpose, a distributed version of the scenario approach to stochastic constrained optimization is adopted, which allows to guarantee by design a predefined robustness level of the obtained solution against uncertainty.
\end{abstract}

\section{INTRODUCTION}

One of the biggest challenges worldwide nowadays is the increasing trend of electrical energy demand, and its impact on the environment. Namely, residential and commercial buildings account for $40 \%$ of the overall energy consumption, [4]. In European countries, out of this $40 \%$, a $76 \%$ fraction is spent for comfort in buildings and heating, ventilation and air conditioning in particular, [3]. This calls for suitable energy management strategies for building thermal control, so as to minimize electric energy consumption, subject to actuation constraints. Studies in this direction include, but are not limited to, [6], [7], [8], [9], [10], [11], [12].

Only a few contributions address the multi-building case, [5], [2]. When dealing with a district network set-up where multiple buildings cooperatively aim at reducing the electrical energy consumption and associated cost while sharing resources, a distributed approach can be adopted. This allows to cope with privacy issues when buildings are willing to cooperate but not to share their consumption profiles. Also, the computational effort involved in the distributed computation of the optimal energy management strategy is reduced if compared with a centralized solution for large scale networks. Stochastic disturbances, e.g., outside temperature, people occupancy, and solar radiation, affecting the building thermal energy request should be accounted for when devising an energy management strategy. Given that a

This work was partially supported by the European Commission under the project UnCoVerCPS, grant number 643921, and by the German Research Foundation (DFG) within the Priority Program SPP 1914 Control of CyberPhysical Systems.

V. Causevic is with Chair of Information-oriented Control, Technical University of Munich, Germany. vedad.causevic@tum. de

A. Falsone, D. Ioli, M. Prandini are with Dipartimento di Elettronica, Informazione e Bioingegneria, Politecnico di Milano, Italy. \{alessandro.falsone, daniele.ioli,maria.prandini\}epolimi.it robust approach where constraints are enforced for all disturbance realizations is often computationally impracticable, and possibly even infeasible, here we opt for a solution which is guaranteed to satisfy constraints for all disturbances except for a set of a-priori defined probability $\varepsilon \in(0,1)$.

In particular, we adopt a data-driven approach to robust optimization that is known in the literature as scenario approach (see e.g. [1]) where robust constraints are replaced by a finite number of constraints, one per observed/extracted value of the uncertain parameter (scenario). The scenario approach has been already proposed for energy management of a building in, e.g., [16], but, to the best of our knowledge, this is the first time that it is used in a multi-building distributed set-up.

Interestingly, in our distributed approach, scenarios can be extracted by each single building separately and do not need to be exchanged among them in order to compute the optimal strategy, thus avoiding the overload of the communication network and preserving the privacy of local information. More importantly, if the number of (local) scenarios is appropriately chosen, then, with high confidence, the optimal strategy for the locally extracted scenarios satisfies the constraints also for unseen realizations of the uncertainty, except for a set of probability smaller than or equal to $\varepsilon$.

The rest of the paper is organized as follows. In Section II the grid-connected district network with multiple buildings, each one equipped with its own chiller plant, and all of them sharing a cooling network, is described. This involves introducing a model of the district components (i.e., building, chiller plant, cooling network) in terms of thermal energy exchange and conversion of thermal energy into the corresponding electrical energy request to the grid. In Section III, we formulate the optimal energy management problem in a stochastic setting so as to account for the disturbances affecting the building thermal evolution. In Section IV a distributed algorithm is described. Finally, a simulation study is shown in Section V and concluding remarks are given in Section VI.

\section{DisTRICT NETWORK MODEL}

The system under consideration is composed of multiple buildings, each one is equipped with its own chiller plant to guarantee a certain level of comfort for its occupants. Moreover, the buildings are connected to a cooling network through which they can exchange cooling energy. The goal is to minimize the overall electrical energy cost for thermal comfort of the building district over a one-day time horizon. 


\section{A. Model of the building}

Following the work in [17], each building is divided into $N_{z}$ thermal zones and the one-day control time horizon is discretized into $M$ time slots of duration $\tau$. The average temperature of zone $j$ is assumed to be set by a low-level controller that is able to adequately track an appropriately defined piece-wise linear reference profile $T_{z, j}(k), T_{z, j}(0)$ being the zone temperature at the beginning of the timehorizon, and $T_{z, j}(k)$ being the temperature at the end of time slot $k$. We collect all building zone temperatures in vector $\boldsymbol{T}_{\boldsymbol{z}}(k)=\left[T_{z, 1}(k) \cdots T_{z, N_{z}}(k)\right]^{\top}$. Let $\boldsymbol{d}(k)$ denote the vector of disturbances (outside temperature, solar radiation, and people occupancy) at the end of time slot $k$.

From [17, Section 2.1], the vector $\boldsymbol{W}_{\boldsymbol{c}}=\left[W_{c}(1) \cdots\right.$ $\left.W_{c}(M)\right]^{\top}$ describing the cooling energy requested by the building to track the temperature set-points of every zone over the time horizon $[1, M]$ can be expressed as

$$
\boldsymbol{W}_{\boldsymbol{c}}=A \boldsymbol{T}(0)+B(\boldsymbol{d}) \boldsymbol{u}+C(\boldsymbol{d})+D \boldsymbol{d},
$$

where $\boldsymbol{T}(0)$ is the building thermal state at the beginning of the time horizon, $\boldsymbol{u}=\left[\boldsymbol{T}_{\boldsymbol{z}}(0)^{\top} \cdots \boldsymbol{T}_{\boldsymbol{z}}(M)^{\top}\right]^{\top}, \boldsymbol{d}=$ $\left[\boldsymbol{d}(0)^{\top} \cdots \boldsymbol{d}(M)^{\top}\right]^{\top}$, and $A, B, C$, and $D$ are suitably defined matrices, with $B$ and $C$ depending on $\boldsymbol{d}$. Note that $W_{c}(k)$ is obtained as $W_{c}(k)=\sum_{j=1}^{N_{z}} W_{c, j}(k), W_{c, j}(k)$ being the cooling energy requested by zone $j$.

\section{B. Model of the chiller unit}

The chiller unit converts electrical energy into cooling energy. Following the derivations in [17, Section 2.2], the electrical energy $W_{c h, \ell}(k)$ needed to produce a certain amount $W_{c h, c}(k)$ of cooling energy during time slot $k$ can be obtained as a piece-wise affine convex approximation of the Ng-Gordon model, [15]:

$$
W_{c h, \ell}(k)=\max \left\{m_{c} W_{c h, c}(k)+q_{c}\right\}
$$

with vectors $m_{c}$ and $q_{c}$ collecting the coefficients of the affine pieces.

\section{Model of the cooling network}

Since the cooling network has a high thermal inertia, it acts as a thermal storage, whose energy content can be described as a first-order dynamical system $S(k+1)=a S(k)-s(k)$, where $S(k)$ is the amount of cooling energy stored, $s(k)$ is the district overall energy exchange $(s(k)>0$ if energy is drawn, and $s(k)<0$ if energy is injected in the network) in the $k$-th time slot, and $a \in(0,1)$ is a coefficient that models energy losses, [17, Section 2.3]. By unrolling the cooling network dynamics over the time horizon $[1, M]$, we get

$$
\boldsymbol{S}=\Xi_{0} S(0)+\Xi_{1} \boldsymbol{s}
$$

where $\boldsymbol{S}=[S(1) \cdots S(M)]^{\top}, \boldsymbol{s}=[s(0) \cdots s(M-1)]^{\top}$, and $\Xi_{0}$ and $\Xi_{1}$ are suitably defined matrices.

\section{THE OPTIMAL ENERGY MANAGEMENT PROBLEM}

The district network is composed of $m$ buildings, each with its own chiller plant. For building $i$, denote with $\boldsymbol{u}_{\boldsymbol{i}}$ the vector containing the temperature set-points of its zones (as appearing in (1)) and with $s_{i}$ the vector containing the contribution of building $i$ to the overall energy exchange of the district in (3) so that $s=\sum_{i=1}^{m} s_{i}$.

The optimal energy management problem consists in finding the values of $\boldsymbol{u}_{\boldsymbol{i}}$ and $\boldsymbol{s}_{\boldsymbol{i}}, i=1, \ldots, m$, so as to minimize the electricity cost along the time horizon $[1, M]$, while satisfying comfort and actuation constraints. Due to the presence of uncertainty, constraints need to be enforced robustly.

Cost function: Let $\boldsymbol{\Psi}=[\Psi(1) \cdots \Psi(M)]^{\top}$ be the vector of the time-varying electric energy price over the reference time horizon $[1, M]$. Then, the electricity cost is given by

$$
J=\sum_{i=1}^{m} J_{i}=\sum_{i=1}^{m} \boldsymbol{\Psi}^{\top} \boldsymbol{W}_{\boldsymbol{c h}, \boldsymbol{\ell}}^{\boldsymbol{i}},
$$

with $\boldsymbol{W}_{\boldsymbol{c h}, \ell}^{\boldsymbol{i}}=\left[W_{c h, \ell}^{i}(1) \cdots W_{c h, \ell}^{i}(M)\right]^{\top}$ being the electric energy needed by the chiller of building $i$ to supply the cooling energy $\boldsymbol{W}_{\boldsymbol{c h}, \boldsymbol{c}}^{\boldsymbol{i}}=\left[W_{c h, c}^{i}(1) \cdots W_{c h, c}^{i}(M)\right]^{\top}$, according to (2). In turn, $\boldsymbol{W}_{\boldsymbol{c h}, \boldsymbol{c}}^{\boldsymbol{i}}$ is given by

$$
W_{c h, c}^{i}=W_{c}^{i}-s_{i},
$$

where $\boldsymbol{W}_{\boldsymbol{c}}^{\boldsymbol{i}}$ is obtained via (1) setting $\boldsymbol{u}=\boldsymbol{u}_{\boldsymbol{i}}$ and $\boldsymbol{T}(0)=$ $\boldsymbol{T}_{\boldsymbol{i}}(0)$ and depends on $\boldsymbol{T}_{\boldsymbol{i}}(0), \boldsymbol{u}_{\boldsymbol{i}}$, and $\boldsymbol{d}$.

Note that $\boldsymbol{d}$ is a global uncertainty vector collecting all disturbances (solar radiation, outside temperature, and people occupancy) affecting all $m$ buildings. Furthermore, note that some of these disturbances (e.g., the outside temperature) may be common to buildings that are close, while others (e.g., people occupancy) may be local to each building.

Comfort constraints: The zone temperatures of each building should be maintained within some comfort range, i.e.,

$$
\boldsymbol{u}_{\boldsymbol{i}}^{\boldsymbol{m i n}} \leq \boldsymbol{u}_{\boldsymbol{i}} \leq \boldsymbol{u}_{\boldsymbol{i}}^{\boldsymbol{m a x}}, \quad i=1, \ldots, m
$$

Actuation constraints related to the cooling network: For each building, a limit is imposed on the amount of energy exchange with the cooling network in each time slot. Also, the energy stored in the network must be non-negative and cannot exceed the network capacity. Therefore

$$
\begin{aligned}
& \left|s_{\boldsymbol{i}}\right| \leq s^{\max }, \quad i=1, \ldots, m, \\
& 0 \leq \Xi_{0} S(0)+\Xi_{1} s \leq S^{\max } .
\end{aligned}
$$

Actuation constraints related to the chiller plant: Constraints are also imposed on the amount of cooling energy required by every building, for each zone, in each time slot of the control horizon. This energy should be positive due to the fact that the chiller can provide cooling energy only. Moreover, the chiller can provide a maximum amount of cooling energy per time slot, which depends on the chiller size and might be different from building to building. This 
translates into

$$
\begin{aligned}
& \boldsymbol{W}_{\boldsymbol{c}, \boldsymbol{j}}^{\boldsymbol{i}} \geq 0 \\
& 0 \leq \boldsymbol{W}_{\boldsymbol{c h}, \boldsymbol{c}}^{\boldsymbol{i}} \leq W_{c h}^{i, \max }
\end{aligned}
$$

for all $i=1, \ldots, m$, where $\boldsymbol{W}_{\boldsymbol{c}, \boldsymbol{j}}^{\boldsymbol{i}}=\left[W_{c, j}^{i}(1) \cdots\right.$ $\left.W_{c, j}^{i}(M)\right]^{\top}$ is the cooling energy request of zone $j$ in building $i, j=1, \ldots, N_{z}^{i}$, over $[1, M]$.

Constraints related to periodicity of the solution: Finally, three additional closure constraints are imposed on the zone temperature set-points, building thermal state, and network energy content so as to obtain a periodic solution if disturbances were periodic. Constraints on the building thermal state are imposed with reference to the average value of the disturbances:

$$
\begin{array}{ll}
\boldsymbol{u}_{\boldsymbol{i}}(M)=\boldsymbol{u}_{\boldsymbol{i}}(0), & i=1, \ldots, m, \\
\boldsymbol{T}_{\boldsymbol{i}}(M)=\boldsymbol{T}_{\boldsymbol{i}}(0), & i=1, \ldots, m, \\
S(M) \geq S(0), &
\end{array}
$$

$\boldsymbol{T}_{\boldsymbol{i}}(M)$ being the thermal state of building $i$ at the end of the horizon. The constraint on the network energy content is useful to avoid depletion of the thermal storage in a myopic finite-horizon perspective that does not account for the day to follow.

Let $X_{i}$ denote the set of constraints defined by (5), (6), (8)-(11) that are local to building $i$ plus the ones involving $\boldsymbol{s}_{\boldsymbol{i}}, i=1, \ldots, m$, in (7) and (12). Let $z$ the optimization vector with components: $\left\{\boldsymbol{u}_{\boldsymbol{i}}\right\}_{i=1}^{m},\left\{\boldsymbol{s}_{\boldsymbol{i}}\right\}_{i=1}^{m}, S(0),\left\{\boldsymbol{T}_{\boldsymbol{i}}(0)\right\}_{i=1}^{m}$. The uncertainty vector $\delta=\boldsymbol{d}$ affects both the cost function $J_{i}$ in (4) and the constraint set $X_{i}$. Introducing an additional optimization variable $\chi_{i}$ per building and enforcing

$$
J_{i}\left(\boldsymbol{u}_{\boldsymbol{i}}, \boldsymbol{s}_{\boldsymbol{i}}, \boldsymbol{d}, \boldsymbol{T}_{\boldsymbol{i}}(0)\right) \leq \chi_{i}
$$

we can replace (4) with $\sum_{i=1}^{m} \chi_{i}$ and move the uncertainty $\delta$ to the constraint set $X_{i}$ through the additional constraint (13), jointly with the upper bound

$$
\chi_{i} \leq \chi_{i}^{\max }
$$

on the maximum electricity cost per building $i$ as dictated by the maximum chiller capacity. Denoting by $\mathcal{X}_{i}$ the constraint set per building $i$ obtained by enlarging $X_{i}$ with (13) and (14), and by $x$ the optimization vector containing $z$ and $\left\{\chi_{i}\right\}_{i=1}^{m}$, we can compactly formulate the energy management problem as

$$
\begin{array}{cl}
\min _{x} & \sum_{i=1}^{m} h_{i}(x) \\
\text { subject to: } & x \in \bigcap_{\delta \in \Delta} \bigcap_{i=1}^{m} \mathcal{X}_{i}(\delta),
\end{array}
$$

where $h_{i}(x)=\chi_{i}$ and we are minimizing the worst-case electricity cost in the cooling district over all admissible disturbances $\delta$ within the uncertainty domain $\Delta$.

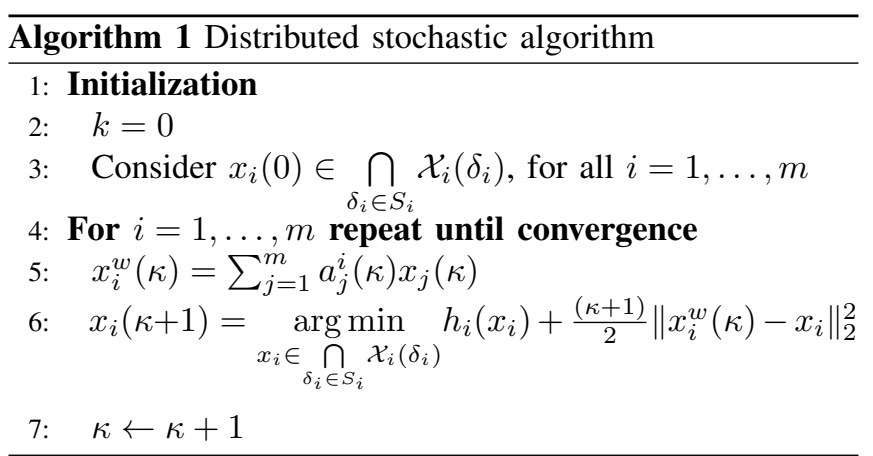

\section{SOlUtion ViA DistribUted ALGORITHM}

Since in our case $\Delta$ is a continuous set, problem (15) is a semi-infinite constrained optimization problem and is, therefore, hard to solve. In order to find a computationally tractable solution, we use a sampled-based method that is known in literature as "scenario approach". To this end, suppose that each building has available $N_{i}$ realizations (scenarios) of the uncertainty parameters and consider the following optimization problem

$$
\begin{array}{cl}
\min _{x} & \sum_{i=1}^{m} h_{i}(x) \\
\text { subject to: } & x \in \bigcap_{i=1}^{m} \bigcap_{\delta_{i} \in S_{i}} \mathcal{X}_{i}\left(\delta_{i}\right),
\end{array}
$$

where $S_{i}$ is the set of local scenarios. If $N_{i}$ satisfies a suitable bound as a function of $\varepsilon$ and $\beta$ as defined in [14], then, with probability at least $1-\beta$, the solution computed from (16) is guaranteed to be feasible for (15) for all uncertainty realizations $\delta$, except for a set of probability at most $\varepsilon$, where $\varepsilon, \beta \in(0,1)$ are selected by the user.

Since we aim at a distributed solution to (16), an iterative approach based on proximal minimization is adopted. In such an approach, an additive quadratic term is added to the local objective function so as to enforce consensus between local decision makers. Problem (16) can then be solved via distributed computations, according to the iterative Algorithm 1.

Algorithm 1 evolves as follows: At iteration $\kappa=0$ each building computes a tentative value for vector $x$. A possible choice is that each building solves a local optimization problem without any additional term. At the generic iteration $\kappa$ in Algorithm 1, each building $i$ computes the weighted average $x_{i}^{w}(\kappa)$ of its own tentative decision and that of its neighbors, with $a_{j}^{i}(\kappa)>0$ only if building $j$ transmits information to building $i$, and $a_{j}^{i}=0$ otherwise (step 5). Then, each building minimizes the sum of its local objective function and a proximal term, which penalizes the distance between its new decision and the previously calculated average (step 6). The relative weight between the two terms in the sum is regulated by the non-decreasing coefficient $(\kappa+1) / 2$, which drives the buildings towards consensus.

Under the following assumptions, the buildings reach asymptotic consensus to one of the minimizers of the optimal 
solution of (16), [14].

Assumption 1: For each $i=1, \ldots, m$, the function $h_{i}(x)$ and constraint set $\mathcal{X}_{i}(\delta)$ are convex in $x$ for any $\delta \in \Delta$, and $\bigcap_{\delta \in S} \mathcal{X}_{i}(\delta)$ is compact, for any finite set $S$ of realizations of $\delta \in \Delta$.

Indeed, since the electrical energy request $W_{c h, \ell}^{i}$ is a piecewise-affine convex function of the cooling energy request $W_{c h, c}^{i}$, and the latter one is linear in the optimization variables, then the sets $\mathcal{X}_{i}(\delta)$ are convex in $x$. The convexity of $h_{i}(x)$ trivially follows from its definition. The compactness condition follows from constraints (5)-(7).

Assumption 2: The constraint $\bigcap_{\delta \in S} \bigcap_{i=1}^{m} \mathcal{X}_{i}(\delta)$ must have a non-empty interior for any finite set $S$ of realizations of $\delta \in \Delta$.

The reader is referred to [14] for additional assumptions on the connectivity of the communication network.

Even though constraints (10) and (11) seem to violate Assumption 2, constraint (10) can be removed by reducing the dimensionality of the decision space using one decision variable for both $\boldsymbol{u}_{\boldsymbol{i}}(0)$ and $\boldsymbol{u}_{\boldsymbol{i}}(M)$. As for constraint (11), it can be used to express $\boldsymbol{T}_{\boldsymbol{i}}(0)$ as an affine function of $\boldsymbol{u}_{\boldsymbol{i}}$ $\left(\boldsymbol{T}_{\boldsymbol{i}}(M)\right.$ is in fact affine in $\boldsymbol{T}_{\boldsymbol{i}}(0)$ and $\boldsymbol{u}_{\boldsymbol{i}}$, see [17] for all the details). The obtained expression can then be used to remove the dependency of all other quantities on $\boldsymbol{T}_{\boldsymbol{i}}(0)$, and, hence, to finally remove constraint (11).

\section{A NUMERICAL CASE STUDY}

In this section we show some numerical examples where the proposed energy management strategy is applied to a three-building network district set-up. For further details the reader is referred to [19].

\section{A. Distributed management of the shared resource}

Consider three medium-size office buildings, each with its own chiller unit, sharing a thermal energy storage. All the three buildings are $20 \mathrm{~m}$ long, $20 \mathrm{~m}$ wide, and $10 \mathrm{~m}$ tall. Each of them is divided into 3 floors, each facade is half glazed and the roof is flat. As for the three chillers, they are all equal and modeled according to the $\mathrm{Ng}$-Gordon formula (see equation (21) in [17]) approximated as in (2) and with maximum cooling energy $W_{c h}^{i, \max }=40 \mathrm{MJ}$. The cooling network has a maximum capacity $S^{\max }=540 \mathrm{MJ}$, a maximum charge/discharge rate $s^{\max }=15 \mathrm{MJ}$ within each time slot, and a loss coefficient $a=0.99$. The control horizon was set to 24 hours and the time slot duration $\tau$ equal to 10 minutes.

The distributed stochastic Algorithm 1 was implemented with $N_{i}=100$ disturbance realizations extracted for each building from the same stochastic disturbance models assuming independence between disturbances. The disturbance realization for the outdoor temperature and short/long-wave radiation are generated as in [18], where the method of functional principal component analysis (FPCA) is employed, although in a different context. The occupancy profiles are instead generated using the model in [13]. We here report them along with their mean value (blue thick line) in Figure 1. The reader should note that past observation of daily disturbances can be used in place of generating them with the aforementioned procedure.

To show the evolution of the algorithm towards consensus, we plot in Figures 2 and 3 the exchanges with the storage $s_{1}, s_{2}, s_{3}$, computed by the three buildings at iterations $\kappa=1$ and $\kappa=50$. In Figure 2 referring to iteration $\kappa=1$, the proxy term appears for the first time and thus imposes a small penalty on the decisions of the buildings about the storage usage. Consequently, the decisions of each building are such that the others mostly charge the storage while the local decision maker uses the stored energy. At iteration $\kappa=$ 50 in Figure 3, the penalty term gains more importance and the buildings let other buildings use the stored energy as well. The algorithm was tested with three types of communication structure between the buildings:

- complete network, where each building is connected to the other two buildings at each iteration;

- connected network, where building 1 is directly connected to buildings 2 and 3, while buildings 2 and 3 communicate indirectly via building 1 , at each iteration;

- time-varying network, where every 3 iterations one building becomes disconnected from the network, to simulate temporary failures in the communication network.

The corresponding number of iterations until convergence is 199, 434 and 722, respectively.

\section{B. Robustness of the obtained solution}

In this further test, we keep the same structure of buildings and storage as previously defined. However, each building has a different chiller now: a small $\left(W_{c h}^{1, \max }=20 \mathrm{MJ}\right)$, medium $\left(W_{c h}^{2, \max }=30 \mathrm{MJ}\right)$, and big chiller $\left(W_{c h}^{3, \max }=40\right.$ $\mathrm{MJ})$. We will refer to the corresponding buildings respectively as building 1 , building 2 and building 3 .
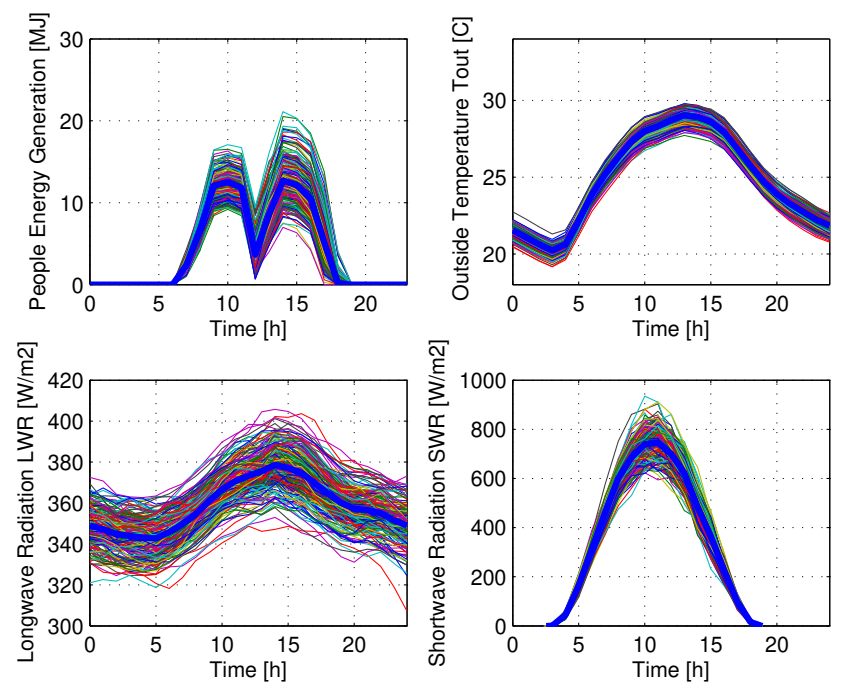

Fig. 1. Disturbance realizations and their mean value (blue thick line) over a one-day horizon. 

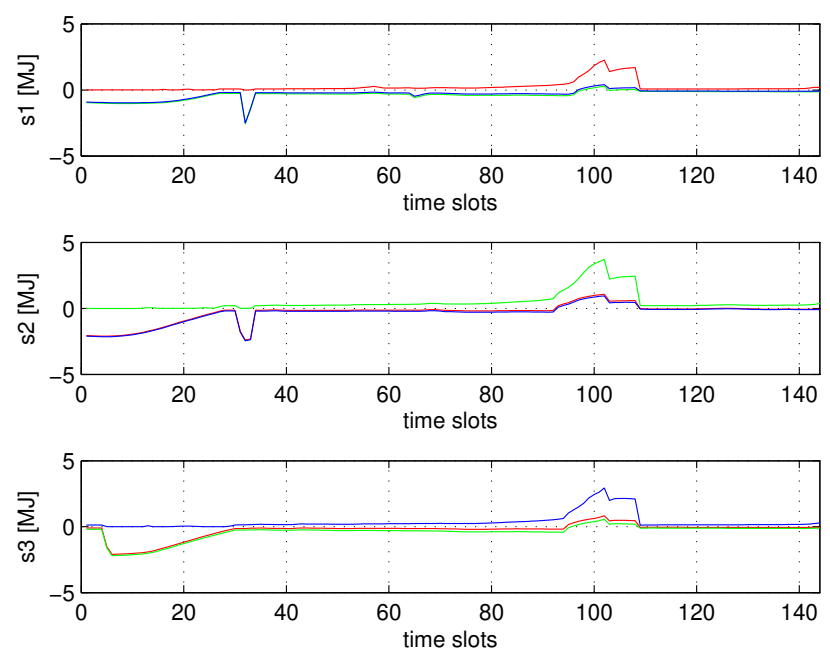

Fig. 2. Energy exchanges with the storage $s_{1}, s_{2}, s_{3}$ (from top to bottom) as computed by building 1 (red), building 2 (green), and building 3 (blue) at iteration $\kappa=1$
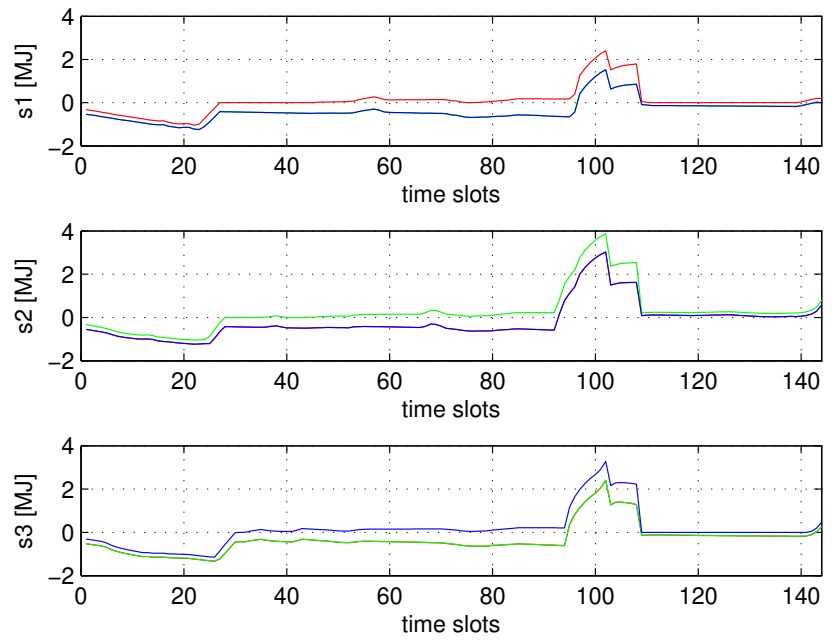

Fig. 3. Energy exchanges with the storage $s_{1}, s_{2}, s_{3}$ (from top to bottom) as computed by building 1 (red), building 2 (green), and building 3 (blue) at iteration $\kappa=50$

The parameters $\varepsilon, \beta$ for the probabilistic guarantees on feasibility in [14] are set to the values: $\varepsilon=0.1, \beta=10^{-6}$. Figure 4 reports the optimal zone temperature set-points for the three floors in the three buildings corresponding to the consensus solution. We see that for each building, the strongest pre-cooling is imposed to the top floor as it is the most exposed to the disturbances. Figure 4 also shows that building 1 (solid lines) tends to keep its zone temperature setpoints lower compared to the other two buildings so as to better exploit the building inertia. This is due to the fact that building 1 has a small chiller. In Figure 5 we see that building 1 is constantly drawing cooling energy from the storage $\left(s_{i}>0\right)$, while building 3 supplies energy to the storage

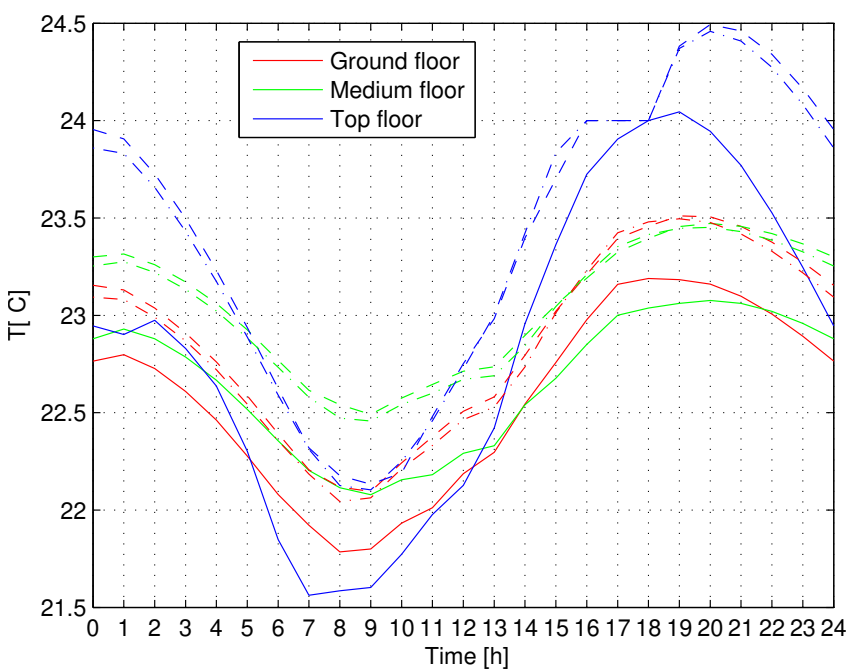

Fig. 4. Optimal zone temperature set-points for the 3 floors of building 1 (solid line), building 2 (dashed line), and building 3 (dash dot line).

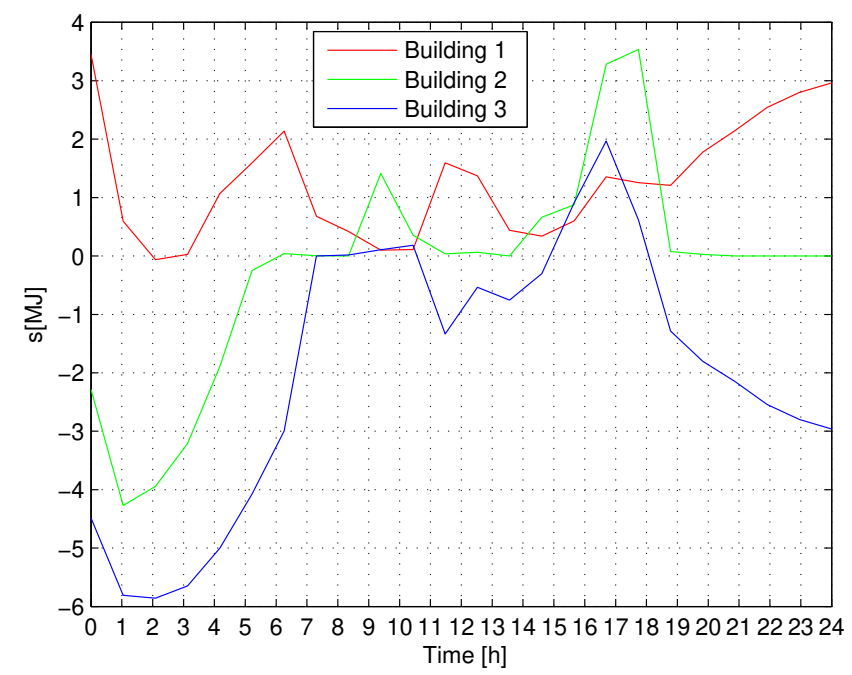

Fig. 5. Energy exchange between the storage and building 1 (red), building 2 (green), and building 3 (blue).

$\left(s_{i}<0\right)$ most of the time. In the late non-occupancy hours (7-12 p.m.) building 3 supplies cooling energy to building 1 through the storage to meet the closure constraints (10)-(12), while building 2 is not participating in the energy exchange with the storage. Overall, it is as if the buildings were sharing their actuation capabilities given by the chillers.

The obtained solution was feasible for $99.8 \%$ of a set of 500 randomly generated disturbance realizations, corresponding to a $0.2 \%$ violation, which is much less than the value of $10 \%$ set for the probability of constraint violation $(\varepsilon=0.1)$. Finally, in Figure 6, one can see the benefit of introducing the cooling network compared to the solution without it. Indeed in the former case the values assumed by the COP are lower than in the latter case. 


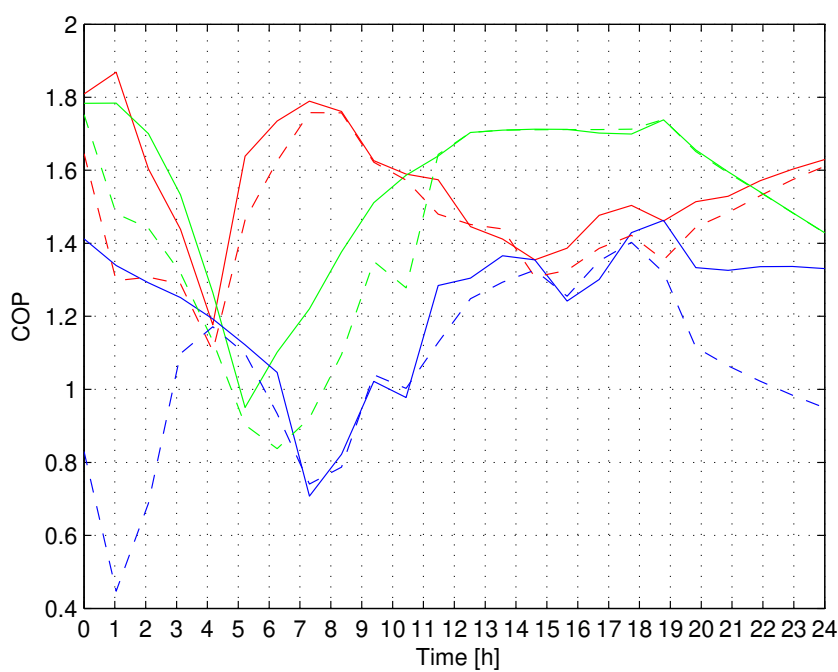

Fig. 6. Average coefficient of performance for chiller 1 (red), chiller 2 (green), and chiller 3 (blue): with storage (solid lines) and without storage (dashed lines).

\section{CONCLUSIONS}

In this paper, distributed stochastic optimization is applied to the problem of optimal energy management of a multibuilding system with reference to thermal control in presence of a shared cooling network that creates the coupling. The algorithm has various nice features: it preserves privacy of information related, e.g, to the building consumption profiles and actuation capabilities; computational load is divided among the buildings; and some degree of robustness against (temporary) failures in the communication network is guaranteed. The framework exploited is very modular, and applicable to different set-ups where, e.g., there are also local storages, heating devices, renewable power generation sources like photovoltaic panels, shared chiller units, etc. Further work is needed to analyze the behavior of building districts with delayed communication links.

\section{REFERENCES}

[1] M.C. Campi, S. Garatti and M. Prandini, The scenario approach for systems and control design, Annual Reviews in Control, vol. 33(2): 149-157, 2009.

[2] D. Ioli, A. Falsone, S. Schuler and M. Prandini, A compositional framework for energy management of a smart grid: a scalable stochastic hybrid model for cooling of a district network, Proceedings of the 12th IEEE International Conference on Control and Automation (ICCA 2016), Kathmandu, Nepal, 2016.

[6] G. Henze, D. Kalz, S. Liu, and C. Felsmann, Experimental analysis of model-based predictive optimal control for active and passive building thermal storage inventory, International Journal of HVAC \& Research, vol. 11, no. 2, pp. 189-214, 2005.
[3] Energy efficiency the right balance between comfort, building performance and sustainability, http://www.siemens.com/energyefficiency.

[4] International Energy Agency, Energy efficiency requirements in building codes, energy efficiency policies for new buildings.

[5] D. Ioli, A. Falsone and M. Prandini, Optimal energy management of a building cooling system with thermal storage: A convex formulation, Proceedings of the 9th IFAC Symposium on Advanced Control of Chemical Processes (ADCHEM 2015), Whistler, British Columbia, Canada, 2015.

[7] Y. Ma, F. Borrelli, B. Hencey, A. Packard, and S. Bortoff, Model predictive control of thermal energy storage in building cooling systems, IEEE Conference on Decision and Control, pp. 392-397, 2009.

[8] J. Siroky, F. Oldewurtel, J. Cigler, and S. Privara, "Experimental analysis of model predictive control for an energy efficient building heating system," Applied Energy, vol. 88, no. 9, pp. 3079-3087, 2011.

[9] Y. Ma, A. Kelman, A. Daly, and F. Borrelli, "Predictive control for energy efficient buildings with thermal storage: Modeling, simulation and experiments," IEEE Control Systems Magazine, vol. 1, no. 1, pp. 45-66, 2012.

[10] F. Oldewurtel, A. Parisio, C. Jones, D. Gyalistras, M. Gwerder, V. Stauch, B. Lehmann, and M. Morari, Experimental analysis of model predictive control for an energy efficient building heating system, Energy and Buildings, vol. 45, no. 2, pp. 15-27, 2012.

[11] K. Deng, Y. Sun, A. Chakraborty, Y. Lu, J. Brouwer, and P. Mehta, Optimal scheduling of chiller plant with thermal energy storage using mixed integer linear programming, American Control Conference, pp. 2958-2963, 2013.

[12] Y. Ma, J. Matusko, and F. Borrelli. Stochastic model predictive control for building HVAC systems: Complexity and conservatism, IEEE Transactions on Control Systems Technology, vol. 23, no. 1, pp. 101116, 2015.

[13] R. M. Vignali, F. Borghesan, L. Piroddi, M. Strelec and M. Prandini. Energy Management of a Building Cooling System with Thermal Storage: An Approximate Dynamic Programming Solution. IEEE Transactions on Automation Science and Engineering, vol. 14, no. 2, pp. 619-633, 2017.

[14] K. Margellos, A. Falsone, S. Garatti, and M. Prandini, Distributed constrained optimization and consensus in uncertain networks via proximal minimization, IEEE Transactions on Automatic Control, pp. 1-16, 2017. Accepted. Available online as an early preview. DOI: 10.1109/TAC.2017.2747505

[15] M. Gordon and K. C. Ng, Cool thermodynamics: The Engineering and Physics of Predictive, Diagnostic and Optimization Methods for Cooling Systems, Cambridge International Science Publishing, 2000.

[16] A. Parisio, D. Varagnolo, M. Molinari, G. Pattarello, L. Fabietti, and K.H. Johansson. Implementation of a Scenario-based MPC for HVAC Systems: an Experimental Case Study, 19th IFAC World Congress, 2014.

[17] D. Ioli, A. Falsone, A. V. Papadopoulos, and M. Prandini. A compositional modeling framework for the optimal energy management of a district network, Journal of Process Control, 2017. DOI:10.1016/j.jprocont.2017.10.005

[18] G. Manganini, A. Falsone, and M. Prandini. A data-based approach to power capacity optimization, Conference on Decision and Control, 2017.

[19] V. Causevic. Energy management in a multi-building setup via distributed stochastic optimization (Master thesis). Politecnico di Milano, 2016. 